\title{
Boundary-controlled barostats for slab geometries in molecular dynamics simulations
}

\author{
C. Gattinoni ${ }^{1, *}$ Sz. Maćkowiak,${ }^{2, \dagger}$ D. M. Heyes, ${ }^{1, \ddagger}$ A. C. Brańka, ${ }^{3,}$ and D. Dini ${ }^{1, \|}$ \\ ${ }^{1}$ Department of Mechanical Engineering, Imperial College London, Exhibition Road, London SW7 2AZ, United Kingdom \\ ${ }^{2}$ Institute of Physics, Poznań University of Technology, Piotrowo 3, 60-965 Poznań, Poland \\ ${ }^{3}$ Institute of Molecular Physics, Polish Academy of Sciences, M. Smoluchowskiego 17, 60-179 Poznań, Poland
}

(Received 1 February 2014; revised manuscript received 27 July 2014; published 3 October 2014)

\begin{abstract}
Molecular dynamics simulation barostat schemes are derived for achieving a given normal pressure for a thin liquid or solid layer confined between two parallel walls. This work builds on the boundary-controlled barostat scheme of Lupkowski and van Swol [J. Chem. Phys. 93, 737 (1990)]. Two classes of barostat are explored, one in which the external load is applied to a virtual regular lattice to which the wall atoms are bound using a tethering potential. The other type of barostat applies the external force directly to the wall atoms, which are not tethered. The extent to which the wall separation distribution is Gaussian is shown to be an effective measure of the quality of the barostat. The first class of barostat can suffer from anomalous dynamical signatures, even resonances, which are sensitive to the effective mass of the virtual lattice, whose value lacks any rigorous definition. The second type of barostat performs much better under equilibrium and wall-sliding nonequilibrium conditions and in not being so prone to resonance instabilities in the wall separation and does not require so many largely arbitrary parameters. The results of exploratory simulations which characterize the dynamical response of the model systems for both dry and wet or lubricated systems using the different barostats are presented. The barostats which have an inherent damping mechanism, such as the ones analogous to a damped harmonic oscillator, reduce the occurrence of large fluctuations and resonances in the separation between the two walls, and they also achieve a new target pressure more quickly. Near a nonequilibrium phase boundary the attributes of the barostat can have a marked influence on the observed behavior.
\end{abstract}

DOI: 10.1103/PhysRevE.90.043302

PACS number(s): 05.10.-a, 83.10.Rs

\section{INTRODUCTION}

Liquids and solids confined between walls have long been studied by means of equilibrium and nonequilibrium molecular dynamics, with periodic boundary conditions only applied parallel to the walls. The simulations can be conducted with the distance between the walls kept constant during the simulation, or where this distance is allowed to vary with time in response to a predetermined normal load or, equivalently, to an external pressure $P_{N}$. It is worth noting that the majority of the experimental work looking at confined fluids in the tribological context, for example, is performed under load control, i.e., the applied pressure is controlled during the experiment rather than the distance between the two surfaces [1,2]. The same boundary conditions also apply to confined solid samples in tribology experiments. Even though the remote load may be applied far from the contact on a molecular distance scale, because of mechanical equilibrium this load is transmitted to the contact zone as a local pressure; therefore, pressure (rather than displacements of the walls) should be controlled as this is, at least in an average sense, the variable that can be directly linked to the external loads in most of the tribological problems of interest (e.g., bearings, engines, artificial joints, and cutting tools). By controlling the applied pressure one can capture both rheology and wall slip behavior of the confined fluid in lubricated contacts and

\footnotetext{
*c.gattinoni@imperial.ac.uk

${ }^{\dagger}$ szymon.mackowiak@doctorate.put.poznan.pl

$\ddagger$ d.heyes@imperial.ac.uk

§branka@ifmpan.poznan.pl

"d.dini@imperial.ac.uk
}

the frictional response of the sliding surfaces in dry and wet conditions; these quantities can be then related to simulations performed at larger scales using continuum approximations (e.g., Refs. [3,4]). Therefore, a facility to control the normal pressure on the confined sample applied at the wall within a molecular dynamics context is desirable. This work proposes and tests a number of new boundary-controlled barostatting schemes.

Andersen [5], derived the first molecular dynamics (MD) barostat, for bulk systems, which was based on an extended Hamiltonian formulation of the equations of motion for the molecules and the cell volume, $V$, which is driven by a fictitious piston. Affine scaling of the molecule's coordinates by the time-dependent factor $V^{1 / 3}$ is employed in this method to achieve a target pressure $P_{B}$ calculated from the virial formula (see Eq. (3.14) in Ref. [5]). The instantaneous pressure of the system fluctuates about this target value, which is a physically realistic feature as it reflects the system's compressibility [6]. Variants of the Andersen method have been developed since [7], as well as a generalization to predefine the pressure tensor, $\underline{P}_{B}$ [8-11], which allows the cell shape to change with time. The Andersen barostat has been applied in dissipative particle dynamics simulations by including a damping term to the equation of motion for the volume [12]. Stochastic or Langevin dynamics equations of motion for the box volume $[13,14]$ and weak-coupling barostat schemes $[15,16]$ have also been used in defining the bulk system barostat. Another formulation of the NPT ensemble has led to new equations of motion for the "piston", redefined as a "shell" particle [17,18]. An alternative approach forces the virial pressure to be held constant by calculating an instantaneous infinite-frequency bulk modulus and adjusting the volume, $V$, to achieve $d P / d t=0$ at all times [19]. 
The bulk system barostats employ uniform or affine scaling of the molecule coordinates and molecular center-of-mass (c.m.) velocities [20,21]. In the boundary confined geometry only one of the diagonal components of the pressure tensor is known in advance, and the other two components parallel to the walls are free to equilibrate to possibly different values if the entrained sample is solid or semisolid. Wang and Fichthorn derived an isothermal-isobaric scheme suitable for confined systems which was based on homogeneous atom position and velocity rescaling, similar to that used for bulk systems [22]. However, in any realistic system the walls cause inhomogeneities in the density and other properties across the gap. Homogeneous or affine scaling of the coordinates and velocities could therefore be considered unphysical. Instead, the desired normal pressure should be achieved through the adjustment of the wall separation so density variations across the gap, and other related quantities, are accommodated in a more realistic manner. This is the procedure adopted here, which is also more consistent with the usual experimental configuration.

In Sec. II a number of confined system barostats are formally presented. Comparisons of the performance of these confined system barostats for a selection of input parameters are then made. Confined liquid film and wearless dry friction systems are considered by molecular dynamics simulations in Secs. III and IV. Conclusions are presented in Sec. V.

\section{THEORY}

The barostats appropriate to boundary confined systems differ markedly in construction to those used for bulk systems as additional issues need to be considered. Figure 1 presents a schematic diagram of a typical confined geometry used

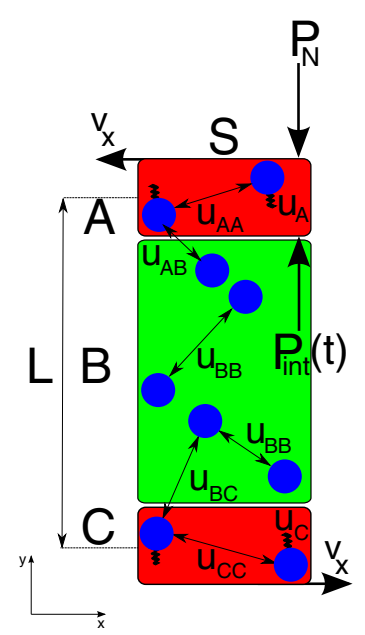

FIG. 1. (Color online) Schematic diagram of the simulation slab geometry. $\mathrm{A}$ and $\mathrm{C}$ are the boundary walls which couple to the normal applied pressure $P_{N}$ and the two walls slide with a velocity $\pm v_{x}$. Region $\mathrm{B}$ is the confined liquid or solid which is allowed to evolve according to classical equations of motion. The pair potentials between atoms in regions $\alpha$ and $\beta$ are denoted by $U_{\alpha \beta}$. The number of atoms in regions $\mathrm{A}, \mathrm{B}$, and $\mathrm{C}$ are denoted by $N_{A}, N_{B}$, and $N_{C}$, respectively. $P_{\mathrm{int}}(t)$ is the time-dependent "internal" pressure which the confined part of the system exerts on the walls. in a molecular dynamics simulation to model a liquid or solid confined between two parallel walls (in the $x z$ plane of the diagram). Three main regions, labeled $\mathrm{A}, \mathrm{B}$, and $\mathrm{C}$ are distinguished. Regions $\mathrm{A}$ and $\mathrm{C}$ represent the solid walls, and region $\mathrm{B}$ is the confined part of the system, which can be a liquid, a solid, or a nonequilibrium steady state that may be a mixture of the two. In the barostatting schemes considered here the atoms in the central region $\mathrm{B}$ follow classical equations of motion. External constraints, such as uniform translation parallel to the walls and a normal force, are applied to regions $\mathrm{A}$ and $\mathrm{C}$ only (or to a subset of the atoms within those regions). The two outer regions, $\mathrm{A}$ and $\mathrm{C}$, are labeled differently because they do not necessarily have to have the same physical characteristics, and external constraints such as uniform translation or an applied normal or shear force could be applied differently to each of them.

The starting point of our discussion is the boundarycontrolled barostat invented by Lupkowski and van Swol [23,24], which we think is the first of its kind. Consider a confined region sandwiched between two walls, $W$, each of mass $M_{W}$. The two walls are treated as solid blocks or monoliths which only couple to the confined molecules through $y$-dependent forces (i.e., each wall has only one degree of freedom). Each wall is subjected to an external normal force, $P_{N} S$, in the $y$ direction, where $P_{N}$ is the applied pressure and $S$ is the cross-sectional area in the $x z$ plane of the periodically repeated simulation cell. The macroscopic equation of motion for the wall-confined material system could be considered for conceptual purposes to be represented by

$$
M_{W} \ddot{y}_{W}=F_{\text {int }}-P_{N} S=\left(P_{\text {int }}-P_{N}\right) S,
$$

where $y_{W}$ is the wall boundary coordinate in the $y$ direction and $F_{\text {int }}=P_{\text {int }} S$ is the net $y$ force on the wall from the molecules in the central region (B). The "internal" pressure, $P_{\text {int }}$ is the net pressure on a wall from the molecules in the central and the other wall regions acting through the pair potential interactions. If the force, $F_{\text {int }}$ were to be derived from a harmonic interaction as a function of $y_{W}$ it would execute simple harmonic motion, and therefore Eq. (1) is referred to as a harmonic oscillator (HO) barostatting scheme. This method has been used in its original form [25] and with the addition of a velocity-dependent damping term to the wall equations of motion [26], where the barostat parallels a damped harmonic oscillator (DHO).

\section{A. Atomistic description of the walls}

A more detailed description of the walls is often required, especially in the context of tribology, where heat conduction and deformation of the confining boundary in sliding conditions, for example, are potentially important aspects of the physics of the system. In fact, many of the previous molecular dynamics simulations of confined liquids and solids (without pressure control however) have been carried out with a discrete atom representation of the boundary wall [27-29] but where the mean position of the wall atoms is fixed. In the following sections, two generically different barostatting procedures for including atomistic detail in the boundary solid wall, which replace the monolithic wall of the Lupowski and van Swol barostat, are proposed. The walls need to be structured, or the 
wall potential should not be a function of the perpendicular direction only, to allow transfer of momentum in the sliding direction.

Two ways of including the atomistic detail of the walls in a barostat scheme are presented here. In the first case, referred to as the virtual-lattice (VL) method, each atom in the wall is tethered to a site of a rigid lattice tracing out part of an ideal crystal. The virtual lattice takes the place of the monolith description of the wall in the Lupkowski and van Swol barostat scheme. The external pressure, $P_{N}$, acts on the virtual-lattice wall lattice sites and therefore only indirectly on the real wall atoms and the rest of the system through the tethering potential. The equation of motion of a real atom, $i$, of mass, $m_{i}$, at $\underline{r}_{i}$ in the wall is $m_{i} \underline{\ddot{r}}_{i}=\underline{F}_{i}+\underline{F}_{T, i}$, where $\underline{F}_{i}$ is the net force on atom $i$ from the other atoms in the wall and from those in the confined region. The force on particle $i$ from the tethered site is $\underline{F}_{T, i}=-\nabla \phi_{T}\left(\left|\underline{r}_{i}-\underline{r}_{0, i}\right|\right)$, where $\phi_{T}$ is the tethering potential and $\underline{r}_{0, i}$ is the location of the relevant virtual-lattice site. As for the Lupowski and van Swol barostat it is necessary to assign a mass, $M_{W}$, to each virtual wall. The wall separation, $L$, is an additional variable which has its own separate equation of motion in the virtual-lattice scheme.

The second approach dispenses with the virtual lattice and tethering potential altogether, and the external force is applied directly to each (real) wall atom or a subset of them. This direct coupling approach is referred to as the direct method (DM). The relationship between the Lupkowski and van Swol barostat to these two other types is illustrated schematically in Fig. 2.

In the direct method the total external force is applied directly to all of the wall atoms ( $N_{W}$ in total) or to a subset, $n_{W}$, typically to the outer layer of atoms in each wall. This option is written formally through the use of the $\epsilon_{i}$ function, applicable to each atom, $i$, in the wall. The function $\epsilon_{i}$ can be 0 or 1 (in which case the barostat piston force is applied to that atom) so $n_{W}=\sum_{j=1}^{N_{W}} \epsilon_{j}$ for each wall. All other atoms in the system execute Newtonian equations of motion. Assuming all wall atoms have the same mass (" $m$ "), the center-of-mass velocity of the system is set to zero in each Cartesian direction at the beginning of the simulation. An equal and opposite net wall force is applied to the designated atoms in the two walls to eliminate overall translation in space of the MD cell. The advantage of the direct-method class of barostat is that there is no ambiguity about the value of the mass used in the equations of motion involved in the coupling procedure. The mass of the wall is just the sum of the masses of the real atoms from which it is composed. This is a clear advantage over the virtual-lattice barostats, where the mass of the virtual-lattice wall is arbitrary and has to be "tuned" to give an optimum system response (see below).

The direct-method barostats can be generalized to include also the relative sliding of the walls to shear the confined region. This is relatively trivial for the virtual-lattice barostats, just by adding at each time step an extra displacement to the motion of the virtual-lattice atoms such that the $x$ component of velocity $\dot{r}_{x, i}$ of each wall atom $i$ becomes $\dot{r}_{x, i} \pm v_{x}$, where $v_{x}$ is the sliding speed. Basically the same operations can be applied in the direct-method case without major consequences, although additional issues need to be considered in a more rigorous implementation of the imposed displacement in the direct-method case as the three regions should be treated as

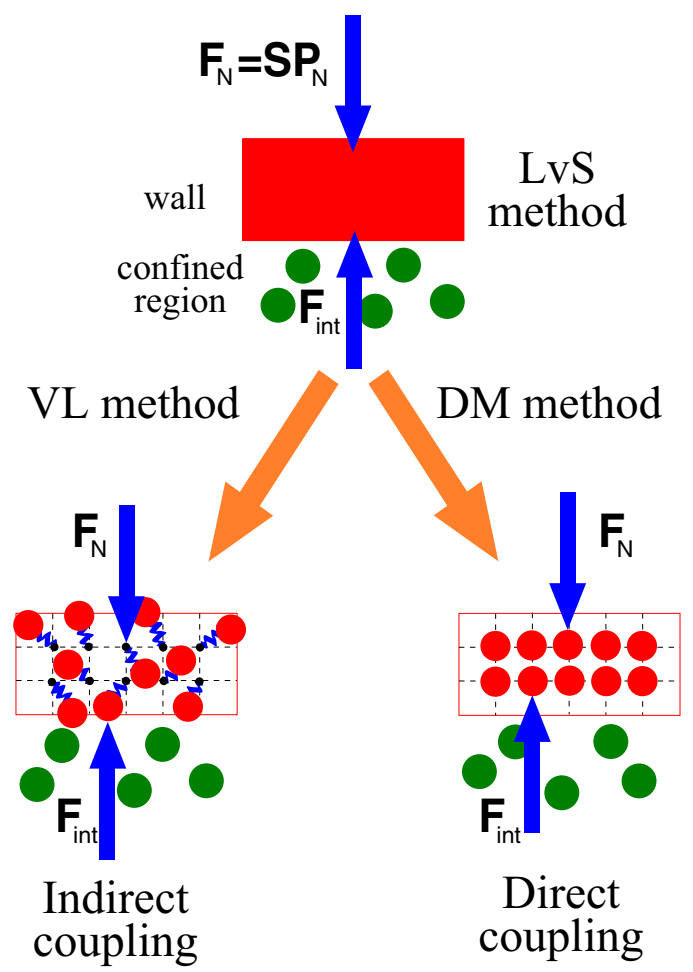

FIG. 2. (Color online) Schematic diagram of the two general classes of barostat considered in this work. The progression from the Lupowski and van Swol barostat (referred to as "LvS" on the top panel) to the virtual lattice (indirect virtual lattice coupling) and direct-method (direct coupling) frameworks is shown in the lower panel. Only one wall is shown.

a single dynamical system, as discussed in Appendices A and $\mathrm{B}$.

Many molecular dynamics simulations reported in the literature have been carried out with a constant time-average wall separation which we refer to as the constant separation (CS) method (e.g., see Refs. [29,30]). The steady-state pressure can be adjusted approximately in advance by bringing the two walls together and then fixing the separation between the walls when a desired value of the pressure has been achieved. It is shown that, on shearing, the steady-state values of the internal pressure and even the physical state of the confined molecules can differ between the CS and a barostatted case, and these two approaches should not be considered to be equivalent in all cases.

The different types of boundary-controlled barostats which can be applied in both the virtual-lattice and direct-method frameworks are described below.

\section{B. Barostats}

\section{Harmonic oscillator}

The first type of barostat is based on a HO approximation. In the virtual wall formulation, this method is closest to the Lupkowski and van Swol barostat, except for the atomistic description of the wall. The governing equation is

$$
\ddot{L}=\left(F_{\text {int }}-F_{N}\right) / M_{W}
$$


where $M_{W}$ is the virtual-lattice wall mass. If the forces in Eq. (2) are, for the sake of simplicity, considered to be harmonic springs, i.e., $F_{\text {int }}=-k L$ and $F_{N}=-k L_{0}$, where $L_{0}$ is the wall separation corresponding to the target pressure, then Eq. (2) reduces to $\ddot{X}+k X / M_{W}=0$, where $X=L-L_{0}$, which is the equation of motion of a harmonic oscillator. Consequently, Eq. (2) is referred to as the VL-HO barostat.

The corresponding direct method, DM-HO, barostat is

$$
m \ddot{y}_{i}=F_{y i}+F_{N i} \epsilon_{i},
$$

which has no free arbitrary parameter. The derivation of the equations of motion (for the case where all wall atoms are barostatted) is presented in Appendix A.

\section{Damped harmonic oscillator}

The second type of boundary barostat is defined for the virtual-lattice case through the equation

$$
\ddot{L}=\left(F_{\text {int }}-F_{N}\right) / M_{W}-\left[c / M_{W}\right] \dot{L},
$$

where $c$ is an arbitrary damping parameter, making this method, in this formulation, a two-free-parameter equation of motion. Note that in the limit $c \rightarrow 0$; Eq. (4) reduces to the virtual-lattice formulations of the $\mathrm{HO}$ scheme of Eq. (2). The other parameters in Eq. (4) have the same meaning as for the HO case of Eq. (2). This is referred to as the VL-DHO barostat. As for VL-HO, the system forces in Eq. (4) when considered to be derived by harmonic springs map onto a damped harmonic oscillator equation, where $\ddot{X}+c \dot{X} / M_{W}+$ $k X / M_{W}=0$ and $X=L-L_{0}$ again. Depending on the values of $M_{W}, k$, and $c$, the relaxation of $X$ (and thus $L$ ) proceeds in an oscillatory, overdamped, or critically damped manner. One of the requirements of a well-performing confined system barostat is that the wall separation should not oscillate unduly to avoid resonance. It is thus reasonable to exclude from our investigation the oscillatory limit which could more easily lead to resonances. This can be achieved by not-too-weak damping in the underdamped case or having near-critical damping. We chose instead to explore the critically damped behavior, which allows us to limit the number of free parameters. Therefore, from here onwards, the DHO barostat should be interpreted as the critically damped case. Note that Pastewka et al. chose a noncritical damping condition in their simulations of boundary confined systems [31]. Critical damping leads to the condition $\omega=\left(k / M_{W}\right)^{1 / 2}=c / 2 M_{W}$, which, when combined with Eq. (4), reduces to

$$
\ddot{X}+2 \omega \dot{X}+\omega^{2} X=0
$$

in the harmonic spring approximation. Therefore with this condition the barostat has been reduced to one-free-parameter, $\omega$, set of equations of motion.

The corresponding direct-method barostat (DM-DHO) can be written as an extension (for $c \neq 0$ ) of Eq. (3) as follows:

$$
m \ddot{y}_{i}=F_{y i}+\left(F_{N i}-c \dot{y}_{i}\right) \epsilon_{i},
$$

where the damping parameter is $c$. A more rigorous derivation of the DM-DHO barostat is given in Appendix B.

\section{Butler-Harrowell}

The last barostat presented here has a foundation that differs substantially from that of the Lupkowski and van Swol barostat, as it is a first-differential-order equation. It was invented by Butler and Harrowell [32] and also has been used subsequently in, for example, Ref. [33]. For the virtual-lattice class this is defined through the equation

$$
\dot{L}=\left(F_{\text {int }}-F_{N}\right) / Q_{P},
$$

where $F_{\text {int }}$ is the total force on the virtual-lattice wall from the tethered real atoms and $F_{N}$ is the force on the virtual wall corresponding to the target pressure applied across the cross-sectional area of the simulation cell (in the $x z$ plane), that is, $F_{N}=P_{N} S$.

The separation between the two walls is $L$ and $Q_{P}$ is an arbitrary damping parameter. Note that the Butler-Harrowell $(\mathrm{BH})$ barostat is based on a first-order differential equation of motion for the virtual-lattice wall separation. This is referred to as the VL-BH barostat. In the corresponding direct-method case, DM-BH, $\dot{L}$ is replaced by the $y$ component of the coordinate of wall atom $i$, and the equation of motion takes the form

$$
\dot{y}_{i}=\left(F_{y i}-F_{N i}\right) \epsilon_{i} / Q_{P},
$$

where $F_{y i}$ is the net $y$ force on atom $i$ from the other atoms in the system, $F_{N i}$ is the external (piston) force per atom in the wall $\left(F_{N} / n_{W}\right)$, and $Q_{P}$ is another damping constant. This is referred to as the DM-BH barostat.

These barostats have been implemented in MD simulations of dry and lubricated systems, exploring the sensitivity of the system response to variation in the free parameters. The free parameter (" $\tau$ ") here is equal to $1 / Q_{p}$ for the VL$\mathrm{BH}$ and DM-BH methods, $1 / M_{W}$ for VL-HO, $c / 2 M_{W}=\omega$ for VL-DHO, and $c$ for the DM-DHO case. For optimum performance, the barostat should give key system properties and their fluctuations, which are sufficiently insensitive to the value of $\tau$ over a reasonably large range of this parameter. In particular, given a target pressure, all barostats and their parameters should lead to the same average value for the time-averaged wall separation, $\langle L\rangle$, for the same physical system. Additionally, a barostat should lead to a stable system and oscillations which do not lead to a resonant state in the wall separation and related properties. The barostat is intended to represent the rest of the solid wall not explicitly included in the atomistic description, whose presence would suppress any such resonances. Large oscillations and resonances are therefore an artifact of the finite-size description of the wall in the simulation. The barostat should respond reasonably rapidly to a new target pressure. A suitable value for the barostat parameter, $\tau$, should be obtainable without extensive preliminary benchmarking of the system's behavior.

Results from tests of the barostats presented in this section can be found in Sec. IV for a solid- and a liquid-entrained system. The solid-entrained system is the most demanding test case for the barostats as it lacks any natural damping which in the liquid-entrained system arises from the compressibility and finite viscosity of the liquid itself. Because of this, we chose to test initially the performance of all the barostats on the solid 
system (Secs. IV B and IV C) and then on a liquid-entrained system in Sec. IV D.

Various versions of the virtual-lattice and direct-method frameworks are used elsewhere in the literature, in conjunction with some of the barostats described here [33-35]. In our description of the barostat we have presented some novel wall-barostat combintations (e.g., VL-HO, VL-DHO, and $\mathrm{DM}-\mathrm{BH}$ ) and we have presented a more rigorous theoretical foundation (both here and in the appendices), which has its roots in statistical mechanics, for all barostat systems.

\section{SIMULATION DETAILS}

Simulations of a solid and a liquid between the walls were carried out. The Lennard-Jones (LJ) potential was used for the pair interactions between the particles,

$$
U_{L J}=4 \epsilon\left[\left(\frac{\sigma}{r}\right)^{12}-\left(\frac{\sigma}{r}\right)^{6}\right],
$$

with parameters corresponding to $\operatorname{argon}\left(\epsilon / k_{B}=120 \mathrm{~K}\right.$ and $\sigma=0.340 \mathrm{~nm}$ ) and truncation distance equal to $2.5 \sigma$. All quantities reported (unless stated) are given in conventional $\mathrm{LJ}$ reduced units, i.e., length in $\sigma$ and time in $\left(m \sigma^{2} / k_{B} T\right)^{1 / 2}$, where $k_{B}$ is the Boltzmann's constant, energy is $\epsilon$, and the atomic mass is $m$ and are all set to unity. The MD calculations were performed with the walls at constant reduced temperature, $T^{*}=k_{B} T / \epsilon=1$. The thermostatting was performed using velocity scaling for the confined solid and the Langevin thermostat in the $z$ direction only for the confined liquid simulations. The confined part of the system was allowed to evolve without a thermostat. De Luca et al. [36] argue that this is the most reliable procedure to produce realistic behavior. The equations of motion were integrated with the leapfrog Verlet algorithm using a time step of $d t=0.001$.

Simulations of the solid entrained system were performed using an in-house code, while simulations of the liquid entrained system were performed using the LAMMPS molecular dynamics package [37]. The solid entrained system was composed of $N=N_{A}+N_{B}+N_{C}$ atoms, where $N_{A}=N_{C}=$ 400 and $N_{B}=1200$, in the geometry shown in Fig. 1. When testing the virtual-lattice method, particles in the walls (regions A and C) were tethered to fixed lattice sites with the anharmonic potential of the following form: $u\left(\vec{r}_{i}\right)=k_{4}\left[\vec{r}_{i}-\right.$ $\left.\vec{r}_{0 i}\right]^{4}+k_{6}\left[\vec{r}_{i}-\vec{r}_{0 i}\right]^{6}$, where $\underline{r}_{0 i}$ is the equilibrium lattice site of atom $i$ [34]. Tethering by anharmonic springs is well known to facilitate kinetic energy equipartition and rapid temperature control [38]. The constants $k_{4}=50$ and $k_{6}=5 \times 10^{3}$ caused root-mean-square displacements at equilibrium which were smaller than $10 \%$ of the mean distance between the wall particles, thus preventing melting of a solid wall and the particles diffusing from region $B$ into regions $A$ and $C$ and vice versa. In the direct-method barostats described in Appendices $\mathrm{A}$ and $\mathrm{B}$, the wall atoms interacted using the $\mathrm{LJ}$ potential in Eq. (9) multiplied by 3 as another way to maintain the solid integrity of the wall. Region B was a solid constructed from a face-centered cubic (FCC) lattice (with the [100] axis along the $x$ direction). The walls (regions $\mathrm{A}$ and $\mathrm{C}$ ) were either amorphous solid or FCC crystalline structures made up of four FCC $(100)$ layers of [5 $\times 5]$ four-atom cells, commensurate with the FCC structure in the $\mathrm{B}$ region formed from 12 layers of
[5 $\times 5$ ] four-atom cells. In the calculations different ABC-slab structure combinations were studied, which are denoted by FFF, ALA, and FLF, where F, A, and L denote FCC crystal and amorphous solid and liquid phases, respectively.

Simulations of the liquid-entrained system were performed to test the direct-method barostats for ca. 50000 atoms in total. Regions $\mathrm{A}$ and $\mathrm{C}$ each contained 18432 atoms of a face-centered cubic (100) lattice at a reduced density $\rho=$ 1.063 with LJ parameters, $\sigma=1$ and $\epsilon=10$ (as above to ensure that the wall remained solid and intact under shear deformation at the highest sliding speeds). Region B contained 11820 liquid atoms with $\epsilon=\sigma=1$, as in Refs. [33,35], and the interactions between the atoms in the wall-liquid boundary region were obtained using the Lorentz-Berthelot mixing rules [39]. The sliding velocity in the $x$ direction was included in the simulations by adding a constant velocity $\pm v_{x}$ at the beginning of the simulation $(t=0)$ to several of the external layers of the two walls. The forces in the $x$ direction for these atoms were set to zero at each time step, thus allowing a constant velocity in the $x$ direction to be maintained when the integration of the equations of motion were performed.

The system relies entirely on thermal coupling to the walls for heat removal. Thermal control of confined systems has been discussed recently by De Luca et al. [36]. An important factor which can influence the thermalization process is the wall-fluid interaction. For example, Khare et al. [40] showed that thermal coupling may be affected by the wetting parameter in such an interaction. Poor coupling is evident as a "jump" in the temperature at the wall-fluid boundary (e.g., see Ref. [41]). Moreover, the slip length depends on the solid-liquid interaction (or the wettability). Pahlavan and Freund [42] showed that the effect of vibrational frequency of solid atoms on the slip behavior is not appreciable, even at high shear rates. In the present simulations with shear the temperature profiles gradually increased with $\epsilon$ above values of ca. 3, without any temperature jump but were essentially independent of barostat method up to the maximum value of $\epsilon$ used in this study $(\epsilon=10)$.

\section{RESULTS AND DISCUSSION}

In this section the various methods for simulating wallconfined systems discussed above are explored computationally. First, the CS scheme is compared with a barostatted system. Then the effects of varying the free parameters in the various barostat schemes are explored in detail. For clarity, the various barostats are treated together within separate virtual lattice and direct coupling method sections because the nature of the parametrization and performance characteristics differ for the two generic classes of barostat.

\section{A. Comparison between constant separation and barostat methods}

Nonequilibrium molecular dynamics simulations were carried out using (a) a constant wall separation and (b) a DM-HO barostat (applied to the two outermost layers of the wall) implemented to compare these two approaches. The initial state was a system which had previously been equilibrated at a pressure, $P=11.85$, with no wall sliding. The confined region 
was a liquid and it was then sheared at a total relative sliding velocity, $v_{x}=0.36$ (with the two walls sliding in opposite directions, $v_{x}= \pm 0.18$ ). Two nonequilibrium steady states are of relevance here [33,35]. In the "plug-slip" (PS) state, the shear gradient is localized in a thin region next to the solid walls, while the rest of the system is hardly flowing and can be viewed as a solid plug to a good approximation. The "central localization" (CL) state is where the shear profile is localized near the center of the gap, while the region $\mathrm{B}$ atoms near the walls take on crystalline order which is in registry with the wall atoms. Figures 3(a) and 3(b) give visualizations of the final state atomic assemblies, which are the PS state for the simulation performed at constant separation [Fig. 3(a)] and the CL for the simulation performed using the DM-HO barostat [left-bottom in Fig. 3(b)] [33]. Figure 3(c) shows the time evolution of the instantaneous value of the wall separation, $L(t)$, using the two procedures. In the constant separation simulation case, $L(t)$ fluctuated about a constant mean value (as it should), whereas in the barostatted system, $L(t)$ increased significantly soon after the start of the simulation. This reflects the dilation induced by an increasing internal pressure in the confined region, which was only able to produce an increased gap width in the barostatted case. The corresponding simulations performed with the DM-BH and DM-DHO barostats produced the same phase transition as the DM-HO barostat. At constant wall separation, the system stayed in the plug-slip state for the entire simulation. The two systems therefore converged to two different values of the wall separation $L$ after $\sim 1500$ reduced time units, with the system at constant separation maintaining the same value of $L$, on average, throughout the simulation and the system barostatted using the DM-HO method showing a dilation of the gap width. The pressure in the system with fixed $L$ grew to 13.5 [see Fig. 3(d)], while it stayed at the target value of 11.85
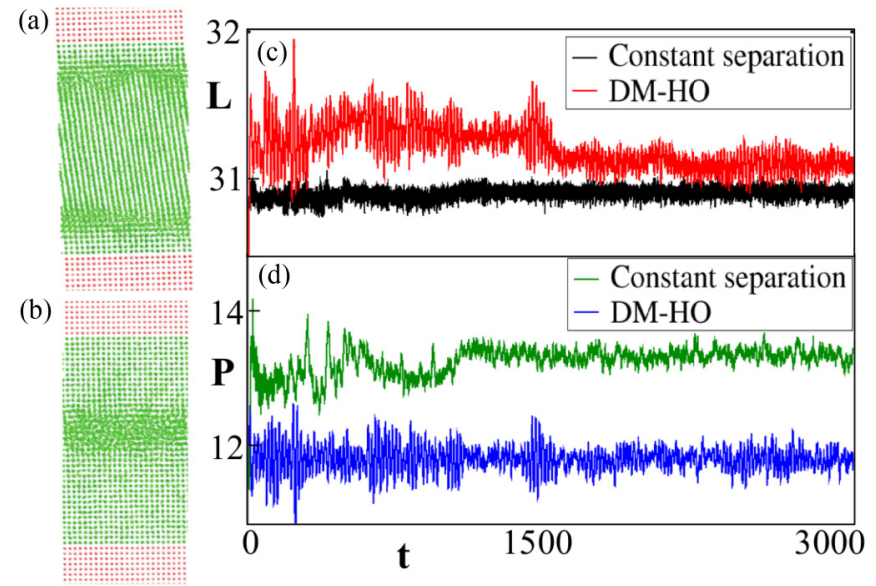

FIG. 3. (Color online) (a) Visualization of the system state at the end of the constant separation simulation. (b) Visualization of the system state at the end of the DM-HO simulation. (c) Evolution of the instantaneous value of the wall separation, $L(t)$, using constant separation (black lower curve) and DM-HO barostat (red upper curve) routes for a liquid lubricated central region. (d) Evolution of the instantaneous value of the pressure in the $z$ direction, $P$, using constant separation (green upper curve) and DM-HO barostat (blue lower curve) routes for a liquid lubricated central region. in the DM-HO system. The inhibition of any gap expansion can be seen therefore to hinder the PS-to-CL nonequilibrium transformation, which relies on a decrease in density of the confined liquid to take place. This demonstrates that a constant film thickness methodology can give qualitatively different results to those of a barostatted simulation of essentially the same system (at least initially).

\section{B. Virtual-lattice simulations}

The wall mass, $M_{W}$, and other damping parameters in the indirect coupling or virtual-lattice barostats need to be given physically realistic values to regulate the degree of a coupling between the virtual lattice and the physical system of interest. If the coupling is too strong or too weak the system can evolve in an unphysical manner or the simulations may become inefficient (too slow to equilibrate) even if the system behaves in a reasonable way over a very long period. There are no formally rigorous formulas which can be used to choose the appropriate values of these parameters. It is known from studies of the bulk thermostat literature (and, by extension, the barostat here) that the "mass" parameter value should be chosen in such a way that the frequency spectrum of the thermostat, (barostat) variable should overlap with that of the system energy fluctuations [43]. This spectral analysis is computationally demanding, which would make it inconvenient for routine use, highlighting the need for a more practical and straightforward criterion to implement. It is shown that in the case of the virtual-lattice and directmethod barostats the statistics of the wall gap length, $L$, variable contains sufficient information to achieve this goal. An investigation of the effects of varying the free barostat parameters is undertaken. The mean wall separation, $\langle L\rangle$, calculated from the three virtual-lattice barostats is shown for FFF and AFA dry contacts over a range of $\tau$ values. The simulations are at equilibrium (i.e., without shearing). The data for the VL-BH, VL-HO, and VL-DHO barostats are shown in Figs. 4-6, respectively. The time evolution of $L$ for three representative values of $\tau$ in each case are shown in the inset frames, while the average wall separation over each simulation, $\langle L(\tau)\rangle$, is shown in the main graph.

For the $\mathrm{BH}$ and $\mathrm{DHO}$ barostats the average separation between the walls $\langle L\rangle$ is constant for an intermediate range of $\tau$ values only. For too-small and too-large values of $\tau$ the mean value of $\langle L\rangle$ differs from the intermediate optimal range of $\tau$ where there is a local plateau $\langle L\rangle$ value. For very large $\tau$ values the system becomes unstable and there is an increasing tendency to produce a resonance effect which can eventually lead to a breakup of the slab. What is outside of this optimal range is not so important, but when $\langle L\rangle$ starts to depend on $\tau$ it helps to establish unequivocally the boundaries of the optimal range. In the case of the $\mathrm{HO}$ barostat, shown in Fig. 5, it is noteworthy that the plateau extends to very large values of $\tau$.

Figure 7 shows the probability distribution of $L(t)$ for the three barostats. The inset shows $P(L)$ against $\left\langle(L-\langle L\rangle)^{2}\right\rangle$. The distribution of $L$ for the $\mathrm{BH}$ and DHO barostats in the stable range of $\tau$ is close to a Gaussian (see the inset) and the time evolution of $L(t)$ shows fairly chaotic behavior, without well-defined oscillation periods, in contrast to the behavior of the HO (compare with Figs. 4 and 6 with Fig. 5). The 


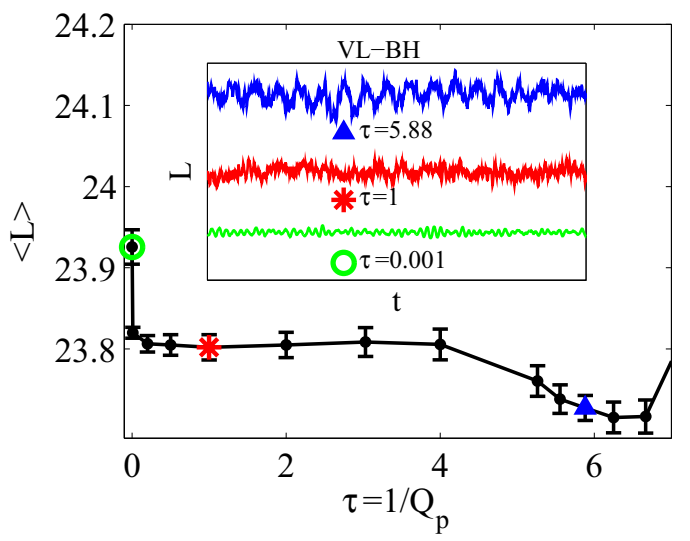

FIG. 4. (Color online) Mean width of the interwall separation, $\langle L\rangle$, for a range of the damping parameter $\tau$ using the VL-BH barostat with $P_{N}=16$ is shown on the main graph. The filled-in black circles with the error bars are the MD results and the lines are to guide the eye. The inset presents the time evolution of $L$, for three representative values of $\tau$, which are also indicated on the main plot with the same symbols.

tendency of the VL-HO is to create an oscillatory response for all values of $\tau$, which may lead to a resonance instability. Our simulations with the solid systems (i.e., FFF and AFA slab geometry) show that in the case of the VL-HO it is hard to find any optimal value regime. There is an oscillatory response with one or a few clearly visible dominant frequencies for all values of $\tau$. If the value of $P_{N}$ is changed such oscillations amplify and can lead to resonance behavior, which is reflected in a standard deviation of $L$ which differs from that of the systems controlled by $\mathrm{BH}$ and $\mathrm{DHO}$ barostats.

The different behavior of the VL-HO barostat observed in the stable region of $\tau$ is also reflected in Fig. 7, where the $P(L)$ distribution is not Gaussian. The same trends were observed for the FFF and AFA types of systems for different $P_{N}$ in the range 6 to 18 reduced units. It was observed that the $\tau$ interval where $\langle L\rangle$ is more or less constant depends only weakly on $P_{N}$. The importance of having Gaussian statistics of an ancillary variable is well established in the thermostat literature [43]. In the case of the barostat it indicates that the wall response is

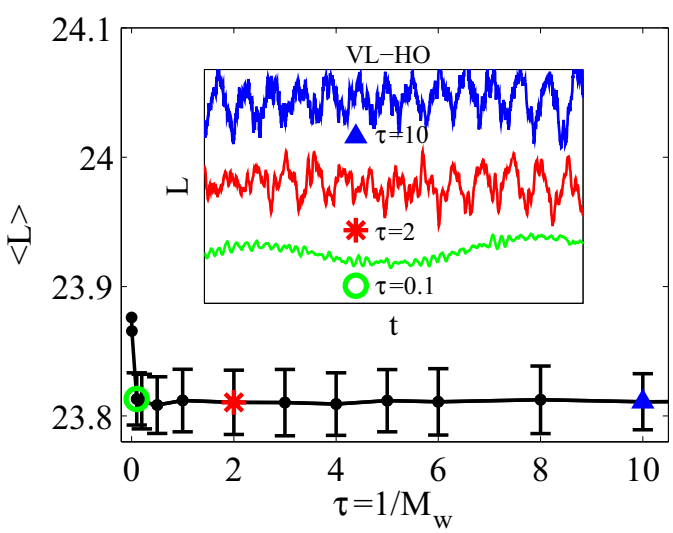

FIG. 5. (Color online) As for Fig. 4 except that the VL-HO barostat was used.

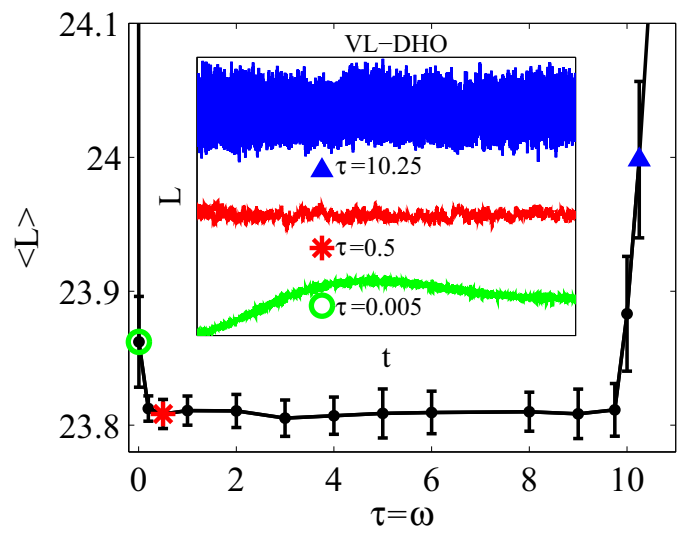

FIG. 6. (Color online) As for Fig. 4 except that the VL-DHO barostat was used. There is no externally imposed shearing.

characterized by a spread of frequencies rather than just a few dominant ones, also clearly seen in the time traces in the insets of Fig. 4-6.

Another measure of the quality or effectiveness of a barostat is its response to a time-dependent load. Figure 8 shows the time dependence of $L$ due to two step changes in $P_{N}$ for the FFF type of slab using the three virtual-lattice barostats.

The target normal pressure was decreased from 10 to 8 and then restored to 10 at times $t=100$ and $t=200$, respectively. The HO response in $L(t)$ is slow and features large highfrequency oscillations superimposed onto the general trend which are consistent with the traces in Fig. 5. The BH scheme takes longer than the DHO barostat to converge to the new steady-state pressure. For the different categories of solid slabs (i.e., FFF and AFA) and for a wide range of applied pressure $6 \leqslant P_{N} \leqslant 18$, the DHO barostat proved superior to the other two approaches as $L(t)$ attained a new steady-state value more quickly on changing the target pressure. Similar behavior was observed for the AFA slab and other combinations of sudden $P_{N}$ change.

To conclude, for the solid confined systems the VL-HO barostat exhibited persistent and regular oscillations in both the time evolution of the wall separation $L(t)$ and the instantaneous system pressure, $P(t)$, which can lead to resonant behavior.

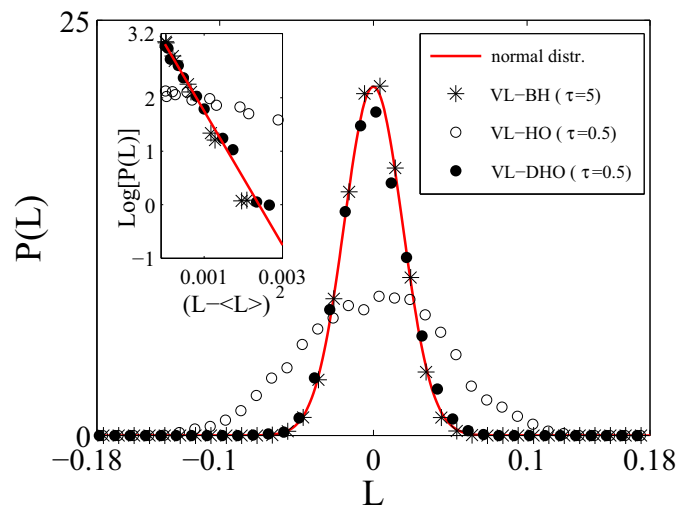

FIG. 7. (Color online) The distribution of the slit width obtained with the indirect coupling (virtual-lattice) barostats. There is no externally imposed shearing. 


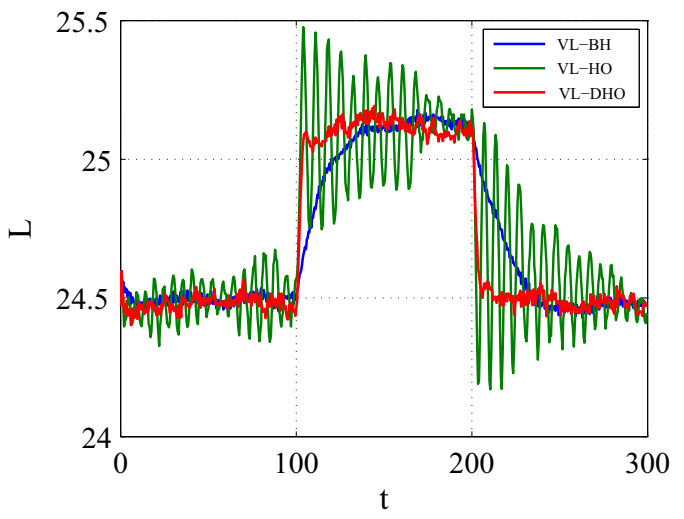

FIG. 8. (Color online) Time evolution of the slab size $L(t)$ generated by three barostats after two sudden changes of the external load. The highly oscillating (green) curve corresponds to the VL-HO barostat, and the (red) curve exhibits sharp step changes in $L$ corresponds to the VL-DHO and the smooth (blue) curve corresponds to the VL-BH. The target pressure is changed from 10 to 8 at $t=100$ and returned to 10 at $t=200$. There is no externally imposed shearing.

The other two barostats showed stable behavior which led to the correct average value of the wall separation, $\langle L\rangle$, for an intermediate range of values of $\tau$ only. For the acceptable values of $\tau$, the VL-DHO is the most efficient and rapid of the three in reaching the target pressure and we consider it to be the best performing of the three virtual-lattice barostats tested.

\section{Direct-method simulations}

In this section the results of corresponding simulations using the direct coupling method are reported. The quantities, $\langle L\rangle, L(t)$, and $P(L)$ were analyzed for the solid entrained region just as for virtual-lattice approach. The DHO barostat, which performed best in its virtual-lattice implementation, and the HO barostat were tested in their direct-method forms. The HO barostat has the advantage of being parameter free, while the DHO scheme needs an optimal value of the parameter $c$ to be determined empirically by a series of preliminary benchmark simulations.

Figure 9(a) shows the mean value of the interwall separation, $\langle L\rangle$, as a function of the frictionlike parameter, $b \equiv c / 2 M_{W}$, defined in Appendix B using the DM-DHO barostat. The results are for a nonsheared system. The inset presents the time evolution of the interwall gap width, $L(t)$, for three representative values of $b$. The selected $b$ are marked by the same enlarged symbols as on the main figure. The $b=0$ case on the figure corresponds to the $\mathrm{HO}$ barostat. Note that there is some difference in $\langle L\rangle$ obtained between the VL-DHO and DM-DHO methods for the same value of $P_{N}$ (e.g., for $P_{N}=10$ the virtual-lattice and direct-method values are 24.5 and 24.9 , respectively). The wall construction and hence its rigidity differed somewhat; in the virtual-lattice case, the wall atoms were tethered and interacted through the standard LJ interaction of Eq. (9), whereas in the direct-method case there was no tethering and the $\mathrm{LJ} \epsilon$ was multiplied by 3 to compensate to sustain the original wall structure during the simulation.

Figures 9(a) and 10 where $b=0$ (i.e., DM-HO) show behavior in $L(t)$ and a distribution $P(L)$ similar to those of the
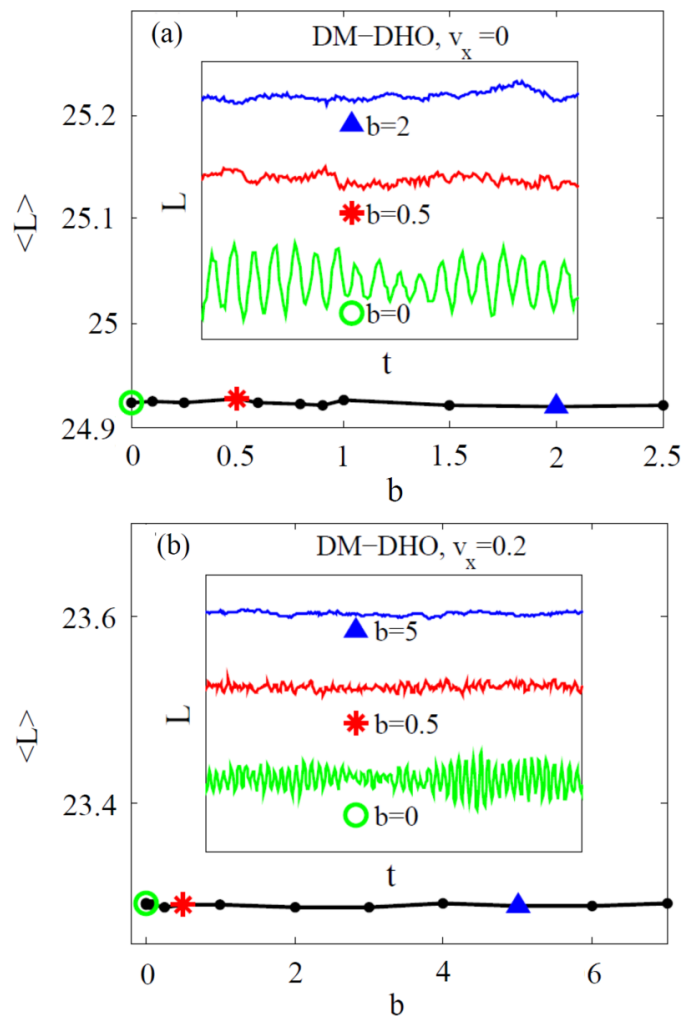

FIG. 9. (Color online) Mean value of the interwall separation, $\langle L\rangle$, as a function of the frictionlike parameter $b\left(\equiv c / 2 M_{W}\right.$ defined in Appendix B) and the DM-DHO barostat of Appendix B. The $b=0$ case corresponds to DM-HO. The filled-in black circles are the MD results obtained for (a) $P_{N}=10$ and $v_{x}=0.0$ and (b) $P_{N}=24$ and $v_{x}=0.2$

VL-HO method presented in Figs. 5 and 7, respectively, in that they both are characterized by regular oscillations in the system properties. Therefore one or a few dominant frequencies determine the time evolution of $L$ which is unwanted in a barostat. Figure 9(a) also shows that a practically constant value of the average wall separation is obtained for a wide range of $b\left(\equiv c / 2 M_{W}\right)$, and the inset demonstrates that variation of $\tau$ produces different oscillatory patterns. The values of $b \approx 0.5$ produce irregular (i.e., nonoscillatory) $L(t)$ and a

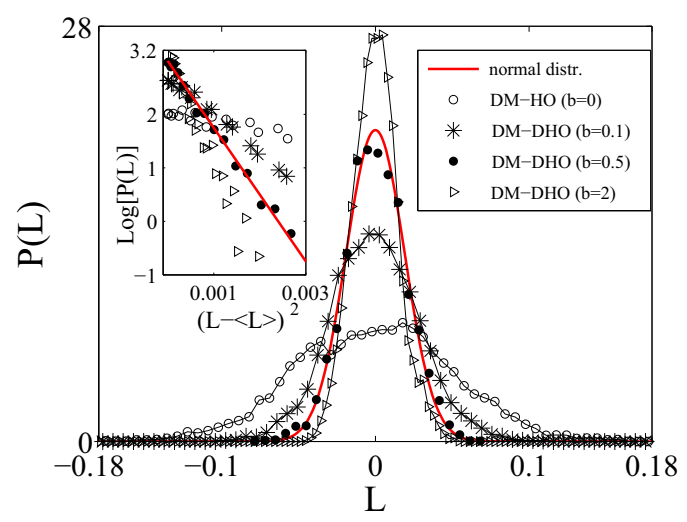

FIG. 10. (Color online) The distribution of the wall separation obtained using the direct-method barostats and selected $b$ values as given in Fig. 9. 

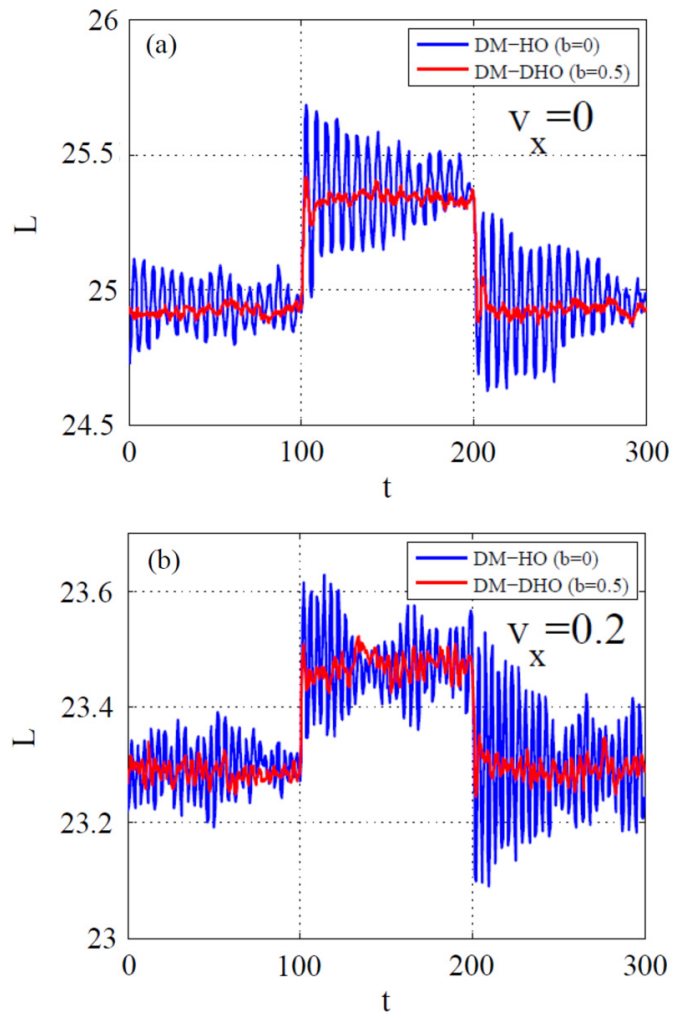

FIG. 11. (Color online) Time evolution of the interwall separation, $L(t)$ generated by the DM-HO and DM-DHO barostats of the form given in Appendices A and B. The highly oscillatory (blue online) curves correspond to the DM-HO case, and the smoother (red) curves to the DM-DHO case. The initial external load pressure was $P_{0}$, which was changed to $P_{1}$ at $t=100$ and returned back to $P_{0}$ at $t=200$. Key: (a) $P_{0}=10, P_{1}=8$, and $v_{x}=0.0$ and (b) $P_{0}=24$, $P_{1}=22$, and $v_{x}=0.2$.

Gaussian $L$ distribution (see Fig. 10) which is satisfactory. For too-large and too-small values of $b$ the distribution tends to deviate from the Gaussian form. It is noteworthy that the value, $b \approx 0.5$, is predicted from the relation $b=\omega$ derived in Appendix B and found empirically to be optimum in the VL-DHO calculations, with $\omega=0.5$, as seen in Figs. 6 and 7 .

Figure 10 shows the distribution of the wall separation obtained using the direct-method barostats for selected $b$ values used in Fig. 9(a). It shows that the DM-HO equations of motion produce a distribution which is not close to Gaussian, which was also the case for the VL-HO in Fig. 7. Figure 11(a) shows the time dependence of $L$ in response to two step changes in $P_{N}$, from 10 to 8 and then from 8 to 10 at times $t=100$ and $t=200$, respectively, for the FFF type of slab. The DM-HO and DM-DHO barostat schemes were used.

As with the corresponding virtual-lattice barostat, DM-HO produces amplified oscillations on top of the general trend which are much greater than those of the DHO scheme. The direct-method barostats in general perform better than their virtual-lattice analogs in regard to the breadth of the optimal range of $\tau$ and their smaller susceptibility to large oscillations, which may lead to unacceptable resonance.

\section{Shear deformation using the direct-method barostats}

Very similar behavior of the direct-method barostats was observed for sheared and unsheared liquid and solid confined samples at different values of the sliding velocities, as may be seen by comparing Figs. 11(a) and 11(b).

The three direct-method barostats were used to simulate equilibrium and sheared liquid confined samples. We decided to test the direct-method barostat only since they provide a wider range of stability for the parameter $\tau$ as well as requiring fewer parameters to tune. In the configuration adopted, only the two layers of atoms at top and bottom of the system (composed of 576 atoms each) experienced the external ("piston") force. These were treated as rigid units. The wall atoms next to the rigid outer layers were fully mobile, executing Newtonian dynamics. Simulations were performed on the system at a reduced pressure of $P_{N}=11.85$ at equilibrium and at a reduced sliding velocity $v_{x}=0.36$ (each wall moving in opposite directions at $v_{x} \pm 0.18$ ). The wall speed value, $v_{x}=0.36$, produces a state which is close to the phase boundary between the PS and CL states on the nonequilibrium phase boundary and a precise control of the pressure is therefore particularly important. The probability distribution of $L$ for the three direct-method barostats was close to Gaussian and the wall separation averages converged to the same value $\langle L\rangle$. The standard deviations of the pressure, $\sigma_{P}$, and slab thickness, $\sigma_{L}$, are summarized in Table I. For all the forms of the direct-method pressure control it was found that the average wall separation at $v_{x}=0.36$ had the same value $\langle L\rangle=31.4$, as evident in Fig. 12. Table I reveals that the amplitude of the oscillations in the pressure and the wall

TABLE I. Standard deviations of the wall separation $L$ and pressure $P$ for the three barostats applied to a lubricated system. $Q_{P}$ is the Butler-Barrowell barostat damping parameter and $c$ is the damping parameter as defined in Eq. (6).

\begin{tabular}{|c|c|c|c|c|c|c|c|c|c|c|c|c|}
\hline & & \multicolumn{11}{|c|}{ Method } \\
\hline & & \multirow[t]{2}{*}{$\mathrm{HO}$} & \multirow[t]{2}{*}{ DHO } & \multicolumn{9}{|c|}{$\mathrm{BH}$} \\
\hline & & & & \multicolumn{9}{|c|}{ Parameter } \\
\hline & & - & $c=1$ & $c=1.5$ & $c=10$ & $c=20$ & $c=50$ & $Q_{P}=1$ & $Q_{P}=20$ & $Q_{P}=50$ & $Q_{P}=100$ & $Q_{P}=200$ \\
\hline \multirow[t]{2}{*}{$v_{x}=0 \mathrm{~m} / \mathrm{s}$} & $\sigma_{L}$ & 0.03 & 0.023 & 0.025 & 0.021 & 0.02 & 0.021 & 2.36 & 0.023 & 0.022 & 0.02 & 0.021 \\
\hline & $\sigma_{P}$ & 1.27 & 1.25 & 1.23 & 1.15 & 1.11 & 1.12 & 11.5 & 1.23 & 1.24 & 1.24 & 1.25 \\
\hline \multirow[t]{2}{*}{$v_{x}=60 \mathrm{~m} / \mathrm{s}$} & $\sigma_{L}$ & 0.42 & 0.2 & 0.14 & 0.09 & 0.05 & 0.04 & 1.17 & 0.05 & 0.04 & 0.035 & 0.034 \\
\hline & $\sigma_{P}$ & 1.94 & 1.45 & 1.39 & 1.23 & 1.2 & 1.23 & 5.22 & 1.35 & 1.33 & 1.34 & 1.33 \\
\hline
\end{tabular}




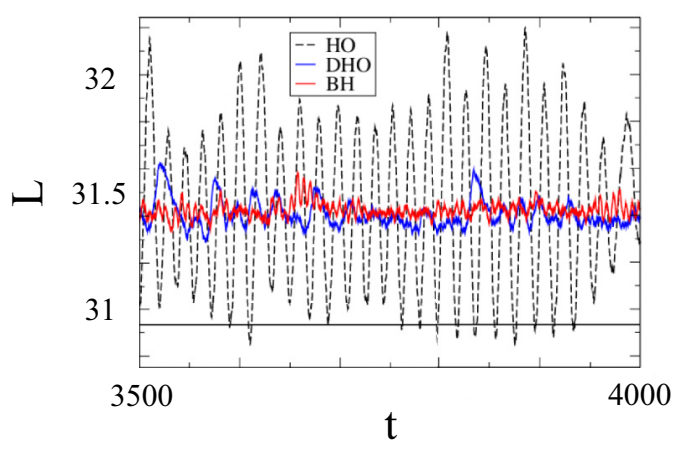

FIG. 12. (Color online) Time evolution of the wall separation $L(t)$ using different direct-method barostatting methods. The (black) oscillatory dashed line is the wall separation obtained by barostatting with the DM-HO scheme. The solid lines show the wall separation for the system using the DM-BH (red) or DM-DHO (blue) schemes The solid horizontal black line is the wall separation observed in the plug-slip phase.

separation is least for the DM-BH and DM-DHO barostats, especially when the system is sheared at $v_{x}=60 \mathrm{~m} / \mathrm{s}$.

Figure 12 shows the time evolution of the wall separation $L(t)$ for the HO, the DHO $(c=10)$, and the BH $(c=50)$ barostats for $v_{x}=60 \mathrm{~m} / \mathrm{s}$, when the system is in the CL state (see Fig. 3). The average wall separation for the same system when it stays in the PS state is also reported as a horizontal solid black line. During the simulations performed with the HO barostat the figure shows that the oscillation amplitude is as large as $1.5 \mathrm{LJ}$ units, and the $\mathrm{HO}$ system was observed to switch frequently between the PS and CL states shown in Fig. 3 during the simulation. However, when the oscillations in the wall separation $L(t)$ are reduced with

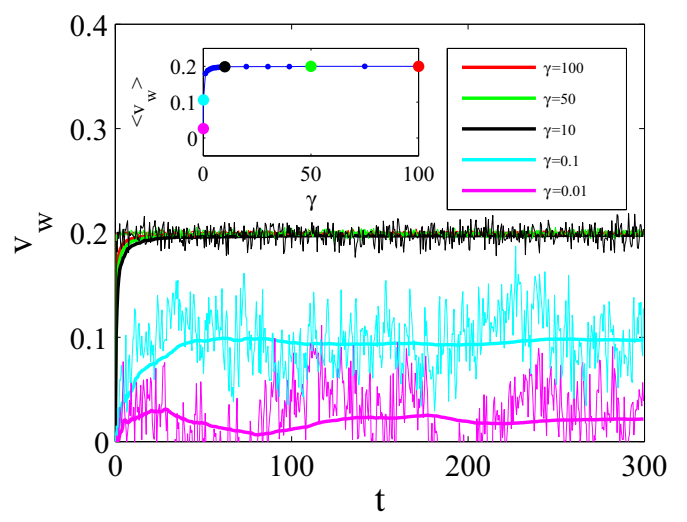

FIG. 13. (Color online) Instantaneous and accumulated mean wall speed (thin and thick lines, respectively) obtained with the DMDHO barostat of Appendix B for different values of the parameter $\gamma$ which are given on the figure. The bottom line (pink) corresponds to $\gamma=0.01$, the middle line (light blue) corresponds to $\gamma=0.1$, and the uppermost lines, which are almost superimposed, correspond to $\gamma=10$ (black), $\gamma=50$ (green), and $\gamma=100$ (red). A FLF type of slab system was used, with $P_{N}=2$ and a target velocity $v_{0}=0.2$. The steady-state wall speed, $\left\langle v_{w}\right\rangle$, achieved as a function of $\gamma$ are shown in the inset. The larger filled circles correspond to the results for $\gamma$ presented in the main graph.

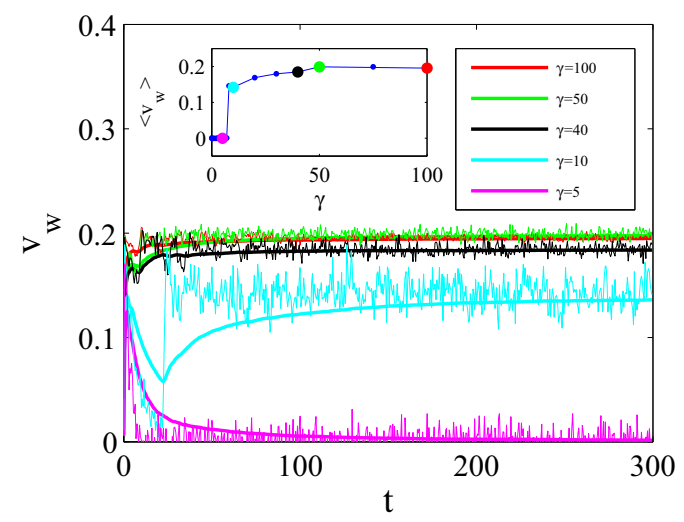

FIG. 14. (Color online) As in Fig. 13 except there is a solid FFF slab, $P_{N}=16$, and $\gamma_{\min } \approx 50$. The bottom line (pink) corresponds to $\gamma=5$ and the middle line (light blue) corresponds to $\gamma=10$. Of the uppermost lines, the lowest one corresponds to $\gamma=40$ (black) and the top two to $\gamma=50$ (green) and $\gamma=100$ (red).

the $\mathrm{DHO}$ and $\mathrm{BH}$ barostats, the system remained in the $\mathrm{CL}$ nonequilibrium state during the simulation. Figure 12 illustrates that the wall separation $L(t)$ for the HO system often overlaps with the wall separation typical of the system in PS configuration (shown in the figure as a solid horizontal line), thus triggering intermittent phase transitions between the PS and CL states. These results demonstrate that close to a (potential) nonequilibrium phase transformation, the type of barostat can have a significant effect on what confined state is produced by the simulation. In the range $10<Q_{P}<200$ the DM-BH barostat gave lower amplitude oscillations than the DM-HO barostat. In the Butler-Harrowell barostat case, a low value of $Q_{P}$ of order unity induced oscillations in the systems which were far greater than in the $\mathrm{HO}$ case, indicating the system was outside its optimal range of $Q_{P}$. A reduction of up to $20 \%$ was obtained in the standard deviation of the DHO pressure for all tested values of the damping parameter, making it a reliable barostatting method.

It is shown in Appendix B that to perform boundary-driven shear of the confined region with the DM-DHO scheme, a driving shear force needs to be applied to the wall particles. This takes the form of $-\gamma\left(\left|\frac{\mathbb{P}_{A}^{x}}{N_{A}}-\frac{\mathbb{P}_{C}^{x}}{N_{C}}\right|-2 p_{0}^{x}\right) \hat{x}$, where $\gamma$ is a shear or friction coefficient and $v_{0}=p_{0}^{x} / m$ is the desired wall speed. This force causes the walls to move with an average velocity, $\left\langle v_{w}\right\rangle=v_{0}$, for a range of $\gamma$-parameter values, greater than a minimum value, $\gamma_{\min }$, as demonstrated in Figs. 13 (liquid entrained) and 14 (solid entrained). The limiting value, $\gamma_{\min }$, depends on the slab properties $v_{0}$ and $P_{N}$. Note that in the case of a liquid entrained system (at low $P_{N}$ value) the walls move even for very small $\gamma$; however, the targeted wall speed $v_{0}$ is not achieved within a reasonable simulation time for $\gamma<\gamma_{\min }$. In the case of solid system a value of $\gamma$ greater than zero is required to start the walls moving and the $\gamma_{\min }$ value is considerably larger than for liquid system. Figures 9(b) and 11(b) were obtained with $\gamma>\gamma_{\min }=100$.

\section{CONCLUSIONS}

Lupkowski and van Swol [23,24] invented a boundarycontrolled barostat for controlling the normal pressure in 
molecular dynamics simulations of liquids confined between planar fluctuating walls, where a wall is represented by a solid block with one degree of freedom [23]. Extensions of this barostat are derived here which incorporate an atomistic description of the wall and where the atoms are tethered to an underlying "virtual" rigid lattice which plays a similar role to the Lupkowski and van Swol barostat wall. This is called the VL method. As a general conclusion, the virtual-lattice barostat closest in analytic form to the Lupkowski and van Swol one is prone to (almost) single-frequency oscillations which can lead to resonances for solids entrained in the gap. A damped version of this method is also proposed here, called VL-DHO, which reduces this tendency, even for entrained solids, subject to the suitable choice of the model damping parameter. Damping is an important prerequisite for a versatile and robust barostat. The new barostats which include some form of damping of the global dynamics of the wall all require at least one damping parameter which needs to be optimized by a preliminary set of simulations.

Another set of barostats is derived which dispenses with the need for a virtual lattice, where the confining force is applied directly to the wall atoms and not to a fictitious sublattice. The relationships between the virtual-lattice and direct coupling schemes are explored analytically and by MD covering a range of damping parameters. It was found that for the virtual-lattice and direct methods a well-performing barostat has a Gaussian distribution of the interwall separation. The damped version of these direct coupling methods showed less sensitivity to wall oscillations over a wider range of optimal damping parameters than the corresponding virtual-lattice case. However, not all values of the damping parameter could be used in practice. This is very much the same restriction as for the so-called $Q$ parameter in the Nosé-Hoover thermostat [43]. In principle you may use any $Q$ value but in practice it is necessary to find an optimal range of $Q$ values, as, if $Q$ is too small, there may be instabilities in the solution and, if too large, very long times will be required to determine system averages with good statistics.

A robust method for imposing a preassigned relative sliding speed between the walls is derived and tested. An additional force is applied to the wall atoms which is proportional to the difference in the instantaneous mean wall velocity difference and a preset target value. It was demonstrated that when the walls are sliding with respect to each other, the nonequilibrium structures formed in the confined region can be sensitive to the type of barostat and indeed whether a barostat is used at all. A fixed wall separation can inhibit the formation of a nonequilibrium structure which requires a shear-induced dilatancy of the interwall gap to form. Moreover, with an undamped barostat, the system can oscillate in time between two nonequilibrium structures. The application of wall-induced shearing of the central region in the direct method involves another parameter which needs to be tuned to achieve a target sliding speed.

The direct-method and virtual-lattice frameworks both have advantages and disadvantages, so which type is preferred largely depends on the application. The direct-method barostats derived here have a sounder theoretical base and are free from inconveniences connected with the need of choosing a damping parameter for the arbitrary virtual-lattice mass. Nevertheless, the virtual-lattice method has been widely used and may have some advantages (e.g., in barostatting molecular systems).

\section{ACKNOWLEDGMENTS}

The work was supported partially by Polish National Science Center Grant No. DEC-2012/05/B/ST3/03255. C.G., D.M.H., and D.D. acknowledge the financial support received from the Engineering and Physical Sciences Research Council (EPSRC) via Platform Grant No. EP/G026114/1. C.G., D.M.H., and D.D. would like to acknowledge the Imperial College High Performance Computing Service for providing the resources to perform the simulations of the liquid entrained system. C.G. and Sz.M. contributed equally to this work.

\section{APPENDIX A: DERIVATION OF THE EQUATIONS OF MOTION OF BAROSTAT, DM-HO}

The DM-HO barostat scheme is derived here for the ABCslab system geometry of Fig. 1. The total number of atoms in the system is $N=N_{A}+N_{B}+N_{C}$ and they interact with each other with the pairwise potential $\phi$. There is no tethering potential. The dynamics of the system is formulated through the following Hamiltonian:

$$
\begin{aligned}
H= & K_{A}+K_{B}+K_{C}+U_{A}+U_{A A}+U_{A B}+U_{B B} \\
& +U_{B C}+U_{C C}+U_{C},
\end{aligned}
$$

where $U_{\alpha \beta}$ is the potential energy interaction between regions $\alpha$ and $\beta$. Indices $\mathrm{A}, \mathrm{B}$, and $\mathrm{C}$ denote a particle or quantity in the regions $\mathrm{A}, \mathrm{B}$, and $\mathrm{C}$, respectively. Thus, the total kinetic energy of the system is $K_{\text {tot }}=K_{A}+K_{B}+K_{C}$ and the potential energy parts of particles in zone A are $U_{A A}=\sum_{i=1}^{N_{A}} \sum_{j=i+1}^{N_{A}} \phi\left(\left|\underline{r}_{i}^{A}-\underline{r}_{j}^{A}\right|\right)$, $U_{A B}=\frac{1}{2} \sum_{i=1}^{N_{A}} \sum_{j=1}^{N_{B}} \phi\left(\left|\underline{r}_{i}^{A}-\underline{r}_{j}^{B}\right|\right)$, and $U_{A}=\sum_{i=1}^{N_{A}} P_{N} \frac{S}{N_{A}} z_{i}^{A}$, where $P_{N}$ is the external load per unit area or the normal pressure acting on the slab. The potential energy of particles in zone $\mathrm{C}$ is obtained by replacing $\mathrm{A}$ and $\mathrm{C}$ (and vice versa) in the above formulas. The three component potential energies which contribute to the potential energy of particles in zone $\mathrm{B}$ are $U_{B B}=\sum_{i=1}^{N_{B}} \sum_{j=i+1}^{N_{B}} \phi\left(\left|\underline{r}_{i}^{B}-\underline{r}_{j}^{B}\right|\right)$, $U_{B A}=\frac{1}{2} \sum_{i=1}^{N_{B}} \sum_{j=1}^{N_{A}} \phi\left(\left|\underline{r}_{i}^{B}-\underline{r}_{j}^{A}\right|\right)$, and $U_{B C}=\frac{1}{2} \sum_{i=1}^{N_{B}} \sum_{j=1}^{N_{C}} \phi\left(\left|\underline{r}_{i}^{B}-\underline{r}_{j}^{C}\right|\right)$. The corresponding equations of motion for the three zones are

$$
\begin{aligned}
& \underline{\dot{r}}_{i}^{A}=\underline{p}_{i}^{A} / m_{i}^{A}, \quad \dot{r}_{k}^{B}=\underline{p}_{k}^{B} / m_{k}^{B}, \quad \underline{r}_{l}^{C}=\underline{p}_{l}^{C} / m_{l}^{C}, \\
& \underline{\dot{p}}_{i}^{A}=\underline{F}_{i}^{A A}+\underline{F}_{i}^{A B}+\underline{F}_{i}^{\mathrm{ex} A}, \\
& \underline{\dot{p}}_{k}^{B}=\underline{F}_{k}^{B A}+\underline{F}_{k}^{B B}+\underline{F}_{k}^{B C}, \\
& \underline{\dot{p}}_{l}^{C}=\underline{F}_{l}^{C C}+\underline{F}_{l}^{C B}+\underline{F}_{l}^{\mathrm{ex} C},
\end{aligned}
$$

where $\underline{F}_{i}^{\mathrm{ex} A}=-P_{N} \frac{S}{N_{A}} \hat{y}$ is the externally applied force to the particle in zone A from the putative piston, $F_{l}^{\operatorname{ex} C}=P_{N} \frac{S}{N_{C}} \hat{y}$ is externally applied force to the particle in the zone $\mathrm{C}$, and $i=1,2, \ldots, N_{A}, k=1,2, \ldots, N_{B}$, and $l=1,2, \ldots, N_{C}$. It is to be noted that the equations for each atom $i$ in the $\mathrm{A}$ and $\mathrm{C}$ wall, in the $y$ direction, take the form of Eq. (3) in the main text.

The total momentum of the whole slab in the DM-HO scheme, $\underline{\mathbb{P}}_{\text {total }}=\underline{\mathbb{P}}_{A}+\underline{\mathbb{P}}_{B}+\underline{\mathbb{P}}_{C}$, is conserved. Also, the center 
of mass of the whole slab is a conserved quantity,

$$
\begin{aligned}
M_{\text {c.m. }} \dot{\mathbb{R}}_{\text {c.m. }} & =\sum_{i}^{N_{A}} m_{i}^{A} \dot{\underline{r}}_{i}^{A}+\sum_{i}^{N_{B}} m_{i}^{B} \underline{\dot{r}}_{i}^{B}+\sum_{i}^{N_{C}} m_{i}^{C} \dot{\underline{r}}_{i}^{C} \\
& =\dot{\mathbb{P}}_{\text {total }}=0 .
\end{aligned}
$$

The Lupkowski and van Swol barostat [23], in fact, can be derived from the above equations. Specifically, the time evolution of the total momentum of the wall zones A and C can be written as follows:

$$
\begin{aligned}
\underline{\mathbb{P}}_{A} & =\sum_{i}^{N_{A}} \underline{\dot{p}}_{i}^{A}=\sum_{i}^{N_{A}}\left(\underline{F}_{i}^{A A}+\underline{F}_{i}^{A B}+\underline{F}_{i}^{\mathrm{exA}}\right) \\
& =\sum_{i}^{N_{A}} \underline{F}_{i}^{A B}-P_{N} S \underline{\hat{y}}=\sum_{j}^{N_{B}} \underline{F}_{j}^{B A}-P_{N} S \underline{\hat{y}}, \\
\underline{\mathbb{P}}_{C} & =\sum_{i}^{N_{C}} \underline{\dot{p}}_{i}^{C}=\sum_{i}^{N_{C}}\left(\underline{F}_{i}^{C C}+\underline{F}_{i}^{C B}+\underline{F}_{i}^{\mathrm{ex} C}\right) \\
& =\sum_{i}^{N_{C}} \underline{F}_{i}^{C B}+P_{N} S \underline{\hat{y}}=\sum_{j}^{N_{B}} \underline{F}_{j}^{B C}+P_{N} S \underline{\hat{y}},
\end{aligned}
$$

Because $\underline{F}_{j}^{B A}$ is the force exerted on particle $j$ in zone B by all the particles from the wall zone $\mathrm{A}$, the force on the wall due to all $N_{B}$ atoms is $\underline{F}_{\text {wall }}^{A}=-\sum_{j}^{N_{A}} \underline{F}_{j}^{B A}$. In the same way, the force on the zone $\mathrm{C}$ is $\underline{F}_{\text {wall }}^{C}=-\sum_{j}^{N_{C}} \underline{F}_{j}^{B C}$. Consequently, within the DM-HO scheme the equations of motion for the walls are as follows:

$$
\begin{aligned}
\dot{\mathbb{P}}_{A} & =\underline{F}_{\text {wall }}^{A}-P_{N} S \underline{\hat{y}}, \\
M_{A} \underline{\mathbb{R}}_{A} & =\sum_{i}^{N_{A}} m_{i}^{A} \dot{\underline{r}}_{i}^{A}=\sum_{i}^{N_{A}} \underline{p}_{i}^{A}=\underline{\mathbb{P}}_{A}, \\
\underline{\mathbb{P}}_{C} & =\underline{F}_{\text {wall }}^{C}+P_{N} S \underline{\hat{y}}, \\
M_{C} \underline{\dot{\mathbb{R}}}_{C} & =\sum_{i}^{N_{C}} m_{i}^{C} \dot{\underline{r}}_{i}^{C}=\sum_{i}^{N_{C}} \underline{p}_{i}^{C}=\underline{\mathbb{P}}_{C},
\end{aligned}
$$

Equations (A9)-(A12) are precisely the equations of motion for the monolith walls proposed by Lupkowski and van Swol (in their notation $\underline{\mathbb{P}}_{A} \equiv \underline{p}_{L}, \underline{\mathbb{R}}_{A} \equiv \underline{r}_{L}, M_{A}=M_{L}, \underline{\mathbb{P}}_{C} \equiv \underline{p}_{R}$, $\underline{\mathbb{R}}_{C} \equiv \underline{r}_{R}, M_{C}=M_{R}$, and $\left.P_{N} S=\alpha_{L}=\alpha_{R}\right)$. With $M_{W}=\bar{M}_{A}$ or $M_{C}, y_{W}=\underline{\mathbb{R}}_{A} \cdot \underline{\hat{y}}$ or $\underline{\mathbb{R}}_{C} \cdot \underline{\hat{y}}$, and $P_{\text {int }}=\underline{F}_{\text {wall }}^{A} \cdot \underline{\hat{y}} / S$ or $F_{\text {wall }}^{C} \cdot \hat{y} / S$ these equations reduce to the form given in $\bar{E} q$. (1).

The correspondence between the present treatment and the Lupowski and van Swol barostat allows us in principle to relate the effective parameters in the Lupowski and van Swol barostat to those in the atomistic description of the wall in DM-HO. In the Lupowski and van Swol barostat the wall mass, $M_{L}$ (and $M_{R}$ ) are unknown parameters and their role is similar to the coupling parameter $\tau$ in barostat schemes VL-HO, VL-DHO, and VL-BH. From the derivation in this appendix it follows that their value should be correlated with the physical mass of the wall, i.e., $M_{L}=M_{A} \sim N_{A} m$. It is noteworthy that the relationship $L=\left(\underline{\mathbb{R}}_{A}-\underline{\mathbb{R}}_{C}\right) \cdot \underline{\hat{y}}$ leads to $\ddot{L}=\left(P_{\text {int }}-P_{N}\right) 2 S / M$, where $M=M_{A}=M_{C}$ and $P_{\text {int }}=\left(\underline{F}_{\text {wall }}^{A}-\underline{F}_{\text {wall }}^{C}\right) \cdot \hat{y} / 2 S$ is like the VL-HO scheme where
$M_{W}=M / 2, F_{\text {int }}=P_{\text {int }} S$ and $F_{N}=P_{N} S$ [see Eq. (4)]. Thus, we can expect a similar performance for Lupowski and van Swol, VL-HO, and DM-HO.

\section{APPENDIX B: THE EQUATIONS OF MOTION OF BAROSTAT, DM-DHO}

The DM-HO equations from Appendix A are extended to incorporate a damping mechanism as follows:

$$
\begin{aligned}
& \underline{\dot{r}}_{i}^{A}=\underline{p}_{i}^{A} / m_{i}^{A}, \quad \underline{\dot{r}}_{k}^{B}=\underline{p}_{k}^{B} / m_{k}^{B}, \quad \dot{\underline{r}}_{l}^{C}=\underline{p}_{l}^{C} / m_{l}^{C}, \\
& \underline{\dot{p}}_{i}^{A}=\underline{F}_{i}^{A A}+\underline{F}_{i}^{A B}+\underline{F}_{i}^{\mathrm{ex} A}-b\left(\frac{\mathbb{P}_{A}}{N_{A}}-\frac{\mathbb{P}_{C}}{N_{C}}\right) \underline{\hat{y}}, \\
& \underline{\dot{p}}_{k}^{B}=\underline{F}_{k}^{B A}+\underline{F}_{k}^{B B}+\underline{F}_{k}^{B C}, \\
& \underline{\dot{p}}_{l}^{C}=\underline{F}_{l}^{C C}+\underline{F}_{l}^{C B}+\underline{F}_{l}^{\mathrm{exC}}+b\left(\frac{\mathbb{P}_{A}}{N_{A}}-\frac{\mathbb{P}_{C}}{N_{C}}\right) \underline{\hat{y}},
\end{aligned}
$$

where the meaning of the quantities and symbols is the same as in the DM-HO scheme. The only difference is the presence of frictionlike terms in the $y$ direction, proportional to the difference between total momentum per particle in zones A and $\mathrm{C}$. These terms are scaled by the constants $b$. Note that if $b=0$ we obtain the DM-HO scheme which therefore can be viewed as a limiting case of a more general DM-DHO scheme. The total momentum and the center of mass of the whole slab, as in the DM-HO scheme, is conserved for $N_{A}=N_{C}$. The wall separation, $L$, is the separation between the centers of mass ( $y$ components) of the A and $\mathrm{C}$ walls. Such a definition is unequivocal also in the case of amorphous walls and is found to be the most natural and useful definition in the present context. The DM-DHO equations with the same definition, $L=\left(\mathbb{R}_{A}-\mathbb{R}_{C}\right) \cdot \hat{y}$, gives $\ddot{L}=\left(P_{\text {int }}-P_{N}\right) 2 S / M-2 b \dot{L}$, where $M=M_{A}=\bar{M}_{C}$ and $N_{A}=N_{C}$, which is Eq. (6) with $b=c / 2 M_{W}=\omega$ and (as in the DM-HO case) $M_{W}=M / 2, F_{\text {int }}=P_{\text {int }} S$, and $F_{N}=P_{N} S$.

The thermostatting procedure of the walls is considered now. In the velocity scaling method, each atomic velocity is multiplied by the factor $\lambda=\sqrt{T_{0} / T}$, which sets the wall temperature to the desired value, $T_{0}$. As the temperature is only controlled in the walls, and noting that $\mathbb{P}_{A}$ and $\mathbb{P}_{C}$ are not conserved quantities, the scaling needs to be performed on the part of the atom's velocity relative to that of the wall as a whole. Hence the scaled momentum of particle $i$ in the wall $\mathrm{A}$ is $\underline{p}_{i}^{A} \lambda-\left(\underline{\mathbb{P}}_{A} \lambda-\underline{\mathbb{P}}_{A}\right) / N_{A}$ and in the wall $\mathrm{C}$ is $\underline{p}_{i}^{C} \lambda-\left(\underline{\mathbb{P}}_{C} \lambda-\underline{\mathbb{P}}_{C}\right) / N_{C}$.

In order to introduce a constant opposite velocity of the walls a shear force is added to Eqs. (B2) and (B4). The resulting equations of motion for a slab system under external load and with the moving walls (with velocity $v_{0}=p_{0}^{x} / m$ ) are

$$
\begin{aligned}
\underline{\dot{r}}_{i}^{A}= & \underline{p}_{i}^{A} / m_{i}^{A}, \\
\dot{p}_{i}^{A}= & \underline{F}_{i}^{A A}+\underline{F}_{i}^{A B}+\underline{F}_{i}^{\mathrm{ex} A}-b\left(\frac{\mathbb{P}_{A}^{y}}{N_{A}}-\frac{\mathbb{P}_{C}^{y}}{N_{C}}\right) \underline{\hat{y}} \\
& +\gamma\left(\left|\frac{\mathbb{P}_{A}^{x}}{N_{A}}-\frac{\mathbb{P}_{C}^{x}}{N_{C}}\right|-2 p_{0}^{x}\right) \underline{\hat{x}}, \\
\underline{\dot{r}}_{k}^{B}= & \underline{p}_{k}^{B} / m_{k}^{B},
\end{aligned}
$$




$$
\begin{aligned}
\underline{\dot{p}}_{k}^{B}= & \underline{F}_{k}^{B A}+\underline{F}_{k}^{B B}+\underline{F}_{k}^{B C}, \\
\underline{\dot{r}}_{l}^{C}= & \underline{p}_{l}^{C} / m_{l}^{C}, \\
\underline{\dot{p}}_{l}^{C}= & \underline{F}_{l}^{C C}+\underline{F}_{l}^{C B}+\underline{F}_{l}^{\mathrm{exC}}+b\left(\frac{\mathbb{P}_{A}^{y}}{N_{A}}-\frac{\mathbb{P}_{C}^{y}}{N_{C}}\right) \hat{y} \\
& -\gamma\left(\left|\frac{\mathbb{P}_{A}^{x}}{N_{A}}-\frac{\mathbb{P}_{C}^{x}}{N_{C}}\right|-2 p_{0}^{x}\right) \hat{x},
\end{aligned}
$$

where $\gamma$ is a shear-rate parameter. The role of the shear force is to produce the desired speed, $v_{0}=p_{0}^{x} / m$, of the walls. If the relative speed of the wall differs from the target value, i.e., $\left|\frac{\mathbb{P}_{A}^{x}}{N_{A}}-\frac{\mathbb{P}_{C}^{x}}{N_{C}}\right|$ differs from $2 p_{0}^{x}$, then the force $\sim \gamma$ starts to act on each wall particle in a way to minimize this difference. In effect, the wall moves with velocity which, when time averaged, is equal to the targeted value, $v_{0}$.
[1] A. Ponjavic, M. Chennaoui, and J. S. S. Wong, Tribol. Lett. 50, 261 (2013).

[2] H. A. Spikes, Lubric. Sci. 18, 265 (2006).

[3] L. Bertocchi, D. Dini, M. Giacopini, M. T. Fowell, and A. Baldini, Tribol. Int. 67, 61 (2013).

[4] A. Strozzi, M. Giacopini, E. Bertocchi, and D. Dini, Proc. Inst. Mech. Eng., Part J: J. Eng. Tribol. 228, 1122 (2014).

[5] H. C. Andersen, J. Chem. Phys. 72, 2384 (1980).

[6] J. R. Ray, Am. J. Phys. 50, 1035 (1982).

[7] W. G. Hoover, Phys. Rev. A 34, 2499 (1986).

[8] M. Parrinello and A. Rahman, Phys. Rev. Lett. 45, 1196 (1980).

[9] M. Parrinello and A. Rahman, J. Appl. Phys. 52, 7182 (1981).

[10] E. Hernández, J. Chem. Phys. 115, 10282 (2001).

[11] S. Melchionna, G. Ciccotti, and B. L. Holian, Mol. Phys. 78, 533 (1993).

[12] S. Y. Trofimov, E. L. F. Nies, and M. A. J. Michels, J. Chem. Phys. 123, 144102 (2005).

[13] D. Quigley and M. I. J. Probert, J. Chem. Phys. 120, 11432 (2004).

[14] S. E. Feller, Y. Zhang, R. W. Pastor, and B. R. Brooks, J. Chem. Phys. 103, 4613 (1995).

[15] R. Faller and J. J. de Pablo, J. Chem. Phys. 116, 55 (2002).

[16] A. Baranyai, J. Chem. Phys. 126, 184111 (2007).

[17] M. J. Uline and D. S. Corti, J. Chem. Phys. 123, 164101 (2005).

[18] M. J. Uline and D. S. Corti, J. Chem. Phys. 123, 164102 (2005).

[19] D. J. Evans and G. P. Morriss, Chem. Phys. 77, 63 (1983).

[20] D. M. Heyes, Chem. Phys. 82, 285 (1983).

[21] C. Braga and K. P. Travis, J. Chem. Phys. 124, 104102 (2006).

[22] J.-C. Wang and K. A. Fichthorn, J. Chem. Phys. 112, 8252 (2000).

[23] M. Lupkowski and F. van Swol, J. Chem. Phys. 93, 737 (1990).

[24] M. Lupkowski and F. van Swol, J. Chem. Phys. 95, 1995 (1991).

[25] H. Takaba, Y. Onumata, and S.-I. Nakao, J. Chem. Phys. 127, 054703 (2007).
[26] L. Zhang, R. Balasundaram, S. H. Gehrke, and S. Jiang, J. Chem. Phys. 114, 6869 (2001).

[27] P. A. Thompson and M. O. Robbins, Phys. Rev. Lett. 63, 766 (1989).

[28] A. Jabbarzadeh, P. Harrowell, and R. T. Tanner, J. Chem. Phys. 125, 034703 (2006).

[29] R. Hartkamp, A. Ghosh, T. Weinhart, and S. Luding, J. Chem. Phys. 137, 044711 (2012).

[30] S. Y. Liem, D. Brown, and J. H. R. Clarke, Phys. Rev. A 45, 3706 (1992).

[31] L. Pastewka, S. Moser, and M. Moseler, Tribol. Lett. 39, 49 (2010).

[32] S. Butler and P. Harrowell, J. Chem. Phys. 118, 4115 (2003).

[33] D. M. Heyes, E. R. Smith, D. Dini, H. A. Spikes, and T. A. Zaki, J. Chem. Phys. 136, 134705 (2012).

[34] J. Petravic and P. Harrowell, J. Chem. Phys. 124, 014103 (2006).

[35] C. Gattinoni, D. M. Heyes, C. D. Lorenz, and D. Dini, Phys. Rev. E 88, 052406 (2013).

[36] S. De Luca, B. D. Todd, J. S. Hansen, and P. J. Daivis, J. Chem. Phys. 140, 054502 (2014).

[37] S. Plimpton, J. Comp. Phys. 117, 1 (1995).

[38] G. Benettin, in Proceedings of the International School of Physics Enrico Fermi, Vol. 15, edited by G. Ciccotti and W. G. Hoover (North-Holland, Amsterdam, 1986).

[39] M. P. Allen and D. J. Tildesley, Computer Simulation of Liquids (Clarendon, Oxford, 1987).

[40] R. Khare, P. Keblinski, and A. Yethiraj, Int. J. Heat Mass Transf. 49, 3401 (2006).

[41] J.-L. Barrat and F. Chiaruttini, Mol. Phys. 101, 1605 (2003).

[42] A. Alizadeh Pahlavan and J. B. Freund, Phys. Rev. E 83, 021602 (2011).

[43] B. L. Holian, A. F. Voter, and R. Ravelo, Phys. Rev. E 52, 2338 (1995). 